\title{
La question des machines, le travail et les savoirs au XIX ${ }^{\mathrm{e}}$ siècle
}

The Machinery Question, Work and Skills in Europe in the $19^{\text {th }}$ century

\section{François Jarrige}

\section{(2) OpenEdition}

\section{Journals}

\section{Édition électronique}

URL : http://journals.openedition.org/artefact/6841

DOI : 10.4000/artefact.6841

ISSN : 2606-9245

\section{Éditeur :}

Association Artefact. Techniques histoire et sciences humaines, Presses universitaires du Midi

\section{Édition imprimée}

Date de publication : 7 janvier 2021

Pagination : 281-315

ISBN : 978-2-8107-0706-5

ISSN : 2273-0753

\section{Référence électronique}

François Jarrige, "La question des machines, le travail et les savoirs au xix ${ }^{\mathrm{e}}$ siècle », Artefact [En ligne], 13 | 2020, mis en ligne le 23 décembre 2020, consulté le 25 décembre 2020. URL : http:// journals.openedition.org/artefact/6841; DOI : https://doi.org/10.4000/artefact.6841

\section{(c) $($ ) $\odot \odot$}

Artefact, Techniques, histoire et sciences humaines est mise à disposition selon les termes de la Licence Creative Commons Attribution - Pas d'Utilisation Commerciale - Pas de Modification 4.0 International. 


\section{La question des machines, le travail et les savoirs au XIX ${ }^{\mathrm{e}}$ siècle}

\section{François Jarrige}

\section{Résumé}

Au xIXe siècle, le développement de nouveaux systèmes techniques de production fondés sur l'emploi croissant de puissantes machines commence à bouleverser les expériences du travail comme les qualifications ouvrières. Ces nouvelles trajectoires s'accompagnent de débats, suivent des chronologies décalées et des chemins variés en fonction des territoires. Les querelles pour fixer le sens du processus et ses enjeux traversent les mondes du travail comme la sphère savante. Pour de nombreux acteurs, le triomphe de la machine menaçait les positions établies, introduisait de nouveaux risques et remettait en cause l'ancienne organisation. Peu à peu néanmoins, s'impose un « âge des machines » qui recompose les rapports sociaux dans un sens jugé bénéfique car il doit alléger les souffrances des travailleurs en accroissant les richesses produites.

\section{Mots-clés}

travail, machines, savoir-faire, conflits, $x \mid x^{e}$ siècle, Europe

99 François Jarrige, « La question des machines, le travail et les savoirs au xixe siècle », Artefact, 13, 2020, p. 281-315. 


\section{The Machinery Question, Work and Skills in Europe in the $19^{\text {th }}$ century}

\section{Abstract}

In the $19^{\text {th }}$ century, the development of new technical production systems based on the increasing use of powerful machines began to change work experiences and worker qualifications. But these new trajectories were accompanied by constant debates in Europe, following staggered chronologies and very different paths depending of territories. The controversies to determine the meaning of the process and its stakes cross the worlds of work as well as the scholarly sphere. For many actors, the triumph of the machine was neither recommended nor really desirable because it threatened established positions, introduced new risks and called into question the Labour organization. Gradually however, a "machine age" is slowly coming into being, that reshapes social relations in a way that is considered beneficial because it should alleviate the sufferings of workers by increasing the wealth produced.

\section{Keywords}

work, machinery, skills, conflict, 19th century, Europe

\section{Note de l'auteur}

Une première version sensiblement différente de ce texte a initialement été publiée en anglais sous le titre "Work, skill and technology", dans THompson Victoria E. (dir.), The Cultural History of Work in the Age of Empire (1800-1920), Arizona State University, Tempe, 2018, p. 85-98. 
u début du $\mathrm{xIx}^{\mathrm{e}}$ siècle, le développement de nouveaux sys1 tèmes techniques de production fondés sur l'emploi croissant 1 de machines commence à bouleverser les expériences du travail comme les qualifications ouvrières. Ces nouvelles trajectoires s'imposent d'abord en Europe, mais elles suivent des chronologies et des chemins très variés et complexes en fonction des territoires. Elles s'accompagnent également de vifs débats pour fixer le sens du processus et ses enjeux. Pour de nombreux acteurs en effet le triomphe de la machine n'était ni souhaitable ni réellement désirable car il menaçait les positions établies, introduisait de nouveaux risques et remettait en cause l'ancienne organisation des gens de métiers ${ }^{1}$. Avant le $\mathrm{XIX}^{\mathrm{e}}$ siècle, la technologie et l'innovation technique restaient des préoccupations secondaires alors que dominait une croissance limitée, essentiellement " organique " et "smithienne ». La technologie des Lumières accordait encore peu de place au grand machinisme, elle restait fondée sur le commerce, l'agencement des composants et l'habileté des artisans ${ }^{2}$. Mais dans l'Europe du XIX ${ }^{e}$ siècle commence à surgir un brouillage croissant des frontières entre les hommes et les machines au service d'un projet d'expansion industrielle, contemporain de l'avènement d'un nouveau type de croissance économique dite "Schumpétérienne ", qui peut être définie comme une augmentation de la production par un développement autonome du progrès technique, un réinvestissement systématique des profits et une rationalisation inédite du travail ${ }^{3}$. La question des machines et des bienfaits à attendre de leur multiplication apparaît dès lors au premier plan lorsque le modèle mécaniste s'impose chez les économistes, savants et hygiénistes qui entendent transformer le corps de l'ouvrier en rouage et en auxiliaire du système productif et de sa croissance ${ }^{4}$.

Alors que s'élabore un nouveau rapport au temps façonné par l'idéologie $\mathrm{du}$ progrès et les révolutions industrielles et scientifiques, l'innovation technologique est de plus en plus considérée positivement, comme le moteur de l'histoire pensée de façon linéaire et évolutionniste ; elle est également de plus en plus identifiée à la sphère de la production et à l'accroissement de la productivité des travailleurs ; elle renvoie désormais à un bouleversement radical censé recomposer les rapports sociaux dans un

\footnotetext{
1. Rule, 1987.

2. Hilaire-Pérez, 2013 ; Halleux, 2009.

3. Verley, 1997, p. 107.

4. Le Roux, 2011 ; Jarrige, 2009.
} 
sens positif en allégeant les souffrances du travailleur et en accroissant les richesses. Pourtant cette évolution est contrebalancée par les doutes sur le caractère destructif du processus. Beaucoup d'observateurs, comme Marx, dénoncent en effet l'insécurité qui s'étend parallèlement au nouveau mode de production capitaliste nécessitant l'existence d'une armée de réserve industrielle pour fonctionner. Alors que les élites célèbrent de plus en plus les inventeurs devenus des héros, et se passionnent pour la mécanique et les savoirs technologiques, le statut de l'innovation se transforme considérablement : "Nouveauté a fini par rimer avec sécurité, routine et tradition avec corruption. Innover devient en soi une sécurité », écrit Liliane HilairePérez $^{5}$. Dès lors se met en place un nouvel imaginaire, où l'innovation va de pair avec l'ordre et le contrôle, rompant avec les anciennes craintes de subversion par la nouveauté. Cet avènement du progrès par la technique remodèle peu à peu le travail et son expérience, les gestes quotidiens comme les règles qui gouvernent la transmission des compétences et l'organisation du travail.

\section{Mécaniser le travail, une nouvelle utopie technologique à l'aube du XIX ${ }^{\mathrm{e}}$ siècle}

Peu nombreuses et peu décisives avant 1800, les machines productives se multiplient ensuite, parallèlement à la réunion des ouvriers dans de grandes manufactures concentrées. Elles deviennent plus visibles et suscitent pléthore de discours et de querelles alors que la force motrice de la vapeur commence à être utilisée, bien souvent en complément des roues hydrauliques ou des anciens manèges de chevaux qui, loin de disparaître, voit leur nombre augmenter dans l'Europe du début du XIx $x^{\mathrm{e}}$ siècle. Les technologies de la vapeur deviennent une source de fierté et de grandeur, et ses promoteurs de véritables héros célébrés dans leur pays respectif, à l'image de James Watt en Angleterre, de Denis Papin en France, ou encore Robert Fulton aux États-Unis ${ }^{6}$. Il convient toutefois de rappeler combien, en 1830, en dehors de quelques régions industrielles anglaises, le système usinier et ses mécaniques perfectionnées restent limités et la division du travail encore très partielle. L'essentiel du travail est toujours réalisé

5. Hilaire-Pérez, 2000 ; pour une histoire intellectuelle du concept d'innovation et ses reconfigurations historiques, cf. Godin, 2015.

6. Berg, 1982 ; MacLeod, 2007. 
manuellement, en recourant à des outils simples, fabriqués localement. Les fameuses " pompes à feu » qui symbolisent le modèle industriel britannique deviennent les machines à vapeur et s'imposent comme le symbole du grand machinisme. Pourtant, elles tardent à se diffuser et de nombreux travaux ont montré la très lente conversion à la vapeur de nombreux territoires industriels européens. La machine à vapeur demeure concurrencée par la persistance des " moteurs animés ", c'est-à-dire le corps des bêtes et des hommes, et les moteurs hydrauliques. En France, comme dans la péninsule italienne, ce n'est pas avant les années 1860-1870 que la puissance des machines à vapeur fixes égale celle des machines hydrauliques, en 1860 celles-ci fournissent encore le double de puissance de la vapeur alors que «le charbon n’a pas encore gagné la partie " ${ }^{7}$. Même dans un pays aussi précocement industrialisé que la Belgique, la vapeur et son système de machines combinées actionnées par un moteur central ne concernent en 1846 que 1000 établissements artisanaux et industriels - certes les plus gros - sur les 114000 que compte alors le pays, alors qu'il existe encore à cette date 2739 moulins à vent, 2633 moulins à eau et 1512 manèges de chevaux dans le pays ${ }^{8}$. Même en Angleterre, l'importance de la vapeur a été relativisée par des travaux soulignant combien, en plein cœur de l'ère victorienne, l'essentiel du travail reste manuel et artisanal'.

En dépit de cette mécanisation lente et progressive, les promesses technologiques circulent de plus en plus en donnant naissance à un messianisme technique qui se retrouve dans de nombreux discours. En Europe, comme aux États-Unis, le culte de la machine s'étend en se nourrissant d'une rhétorique morale identifiant les techniques à un monde ordonné et pacifié. Dans le monde anglo-américain en particulier, la technologie s'affirme comme l'un des piliers de l'avenir et de la puissance de la nation ${ }^{10}$. OutreAtlantique, de nombreux auteurs, souvent venus d'Europe, deviennent ainsi des prophètes enthousiastes de l'âge mécanique en associant intimement les prouesses techniques de l'ère industrielle et un horizon d'abondance et d'émancipation ${ }^{11}$. C'est par exemple le cas de John Etzler, un

7. Alain Leménorel, Gabriel Désert, Philippe Dupré, Yannick Lecherbonnier, Emmanuelle Réal, 1993 ; Jarrige et Vrignon, 2020.

8. Van Neck, 1979, p. 598 et suiv. ; Von Tunzelmann, 1978.

9. Samuel, 1977 ; Malm, 2016.

10. Nye, 1994, p. 46.

11. Marx, 2000 [1964]. 
émigrant allemand installé aux États-Unis, et auteur en 1833 d'un livre où il annonce l'avènement du paradise within the reach of all men without labor, by powers of nature and machinery ${ }^{12}$. Etzler, fortement imprégné des théories du réformateur français Charles Fourier, voyait l'Amérique comme un nouvel Eden où l'homme, être rationnel, pourrait construire, grâce aux machines, une société pacifiée et heureuse, à mille lieues des pesanteurs des monarchies autocratiques qui dominaient le continent européen. Il dépose lui-même de nombreux brevets ${ }^{13}$. L'inventeur devient un héros célébré et le statut de l'invention se transforme avec la généralisation du modèle du brevet. Les législations nationales visant à protéger les inventeurs en leur reconnaissant un droit moral et sacré sur leur découverte apparaissant en effet partout, quoiqu'à des rythmes variables selon les pays.

Parallèlement à la multiplication des mécaniques qui recomposent les savoirs et les qualifications ouvrières, apparaissent aussi de multiples théories des machines, c'est-à-dire des approches analytiques de leur fonctionnement, un corpus de savoirs visant à standardiser leur fabrication et accroître leur nombre et leur rendement ${ }^{14}$. Dès 1724 , dans son Théâtre des machines, l'Allemand Jacob Leupold définissait déjà la machine comme " une disposition artificielle au moyen de laquelle on peut mouvoir quelque chose avec économie de temps et de force ". Mais c'est surtout entre 1800 et 1850 que les tentatives de définitions se multiplient et s'affinent. Au milieu du XIX ${ }^{\mathrm{e}}$ siècle, la mécanique industrielle est devenue un domaine du savoir délimité, avec sa théorie des moteurs, des roues dentées ou de la transmission de l'énergie. Le groupe des ingénieurs et techniciens tend alors à s'autonomiser et à conquérir une position sociale de plus en plus importante, quoique variable selon les pays. Alors qu'en Grande-Bretagne dominent les «ingénieurs civils », en France s’imposent les grands corps qui forment une véritable " technocratie d'État " dotée d'une autorité et d'un pouvoir très importants ${ }^{15}$. Ces groupes deviennent des acteurs de premier plan de l'introduction des mécaniques et de leur justification. Portés par l'idéal méritocratique, convaincus d'être les agents du progrès, les ingénieurs exercent une influence croissante dans la définition du travail productif, bien au-delà du monde des entreprises et des

12. Etzler, 1833.

13. Claeys, 1986.

14. Daumas, p. 92-93.

15. Belhoste et Chatzis, 2007. 
ministères. Les échanges entre savants, hommes pratiques, fabricants ou chefs d'atelier, entre les laboratoires et l'usine, tendent à s'accentuer et à stimuler l'essor de la construction mécanique tout en modifiant l'organisation de l'industrie. Le mot " technologie ", d'abord conçu à l'époque des Lumières comme la science des opérations artisanales, tend d'ailleurs à se transformer dans ce contexte pour désigner de plus en plus la « science des machines ", un corpus de savoirs visant à optimiser la productivité des mécaniques, leur capacité à accroître les profits par une réforme du travail et de son organisation ${ }^{16}$.

\section{Le choc des machines : le cas des tondeuses mécaniques de draps autour de 1830}

Alors qu'elles sont encore peu nombreuses et assez peu spectaculaires, les machines productives commencent à pénétrer dans les ateliers au cours de la première moitié du XIX ${ }^{e}$ siècle, notamment dans le secteur textile, en provoquant leur lot d'interrogations et de plaintes. Dans le textile, les métiers à tisser et à filer, les mécaniques pour tondre les draps plus ou moins automatiques, se multiplient en suscitant craintes et débats et même, parfois, des émeutes. Lorsqu'elles sont introduites, les nouvelles machines sont perçues par beaucoup comme des " tueuses de bras ", les ouvriers les accusent de leur " couper les bras " et refusent d'abandonner leurs compétences incorporées dans le corps pour devenir des automates mécaniques. Tout au long du premier $\mathrm{XIX}^{\mathrm{e}}$ siècle, la mécanisation du travail est ainsi jalonnée de conflits et de tensions lorsqu' elle menace les positions établies et l'organisation du travail. Les plus célèbres de ces émeutes sont celles du luddisme anglais des années 1811-1812, du nom du mythique général Ned Ludd, meneur imaginaire de la révolte. Les Luddites s'opposent notamment aux métiers textiles accusés de baisser les coûts de production et les salaires ; les tondeurs de draps du Yorkshire notamment s'opposent aux tondeuses mécaniques dans la phase finale de production des draps et l'écho de ces violences est important sur le continent européen ${ }^{17}$. Le cas du travail des tondeurs de draps européens, travaillant dans de petits ateliers au moyen

16. Guillerme et Sebestik, 2007 [1968] ; Carnino, 2010.

17. Thompson, 1991 ; Randall, 1991 ; Binfield (dir.), 2004 ; Bourdeau, Jarrige et Vincent, 2006. 
de forces à tondre et d'un savoir-faire complexe, mérite qu'on s'y arrête. C'est en effet l'une des premières opérations productives à être mécanisée massivement au moyen de grandes machines automatiques, et à l'échelle de l'Europe, entre 1810 et 1840 . Comme l'écrivait Gérard Gayot : « en quelques années un petit monde d'une richesse extraordinaire en mots et en gestes, en habits de travail et en habitudes de vie, en sources morales et intellectuelles, s'écroula ${ }^{18}$ ".

\section{Tableau comparatif simplifié des trois procédés}

\begin{tabular}{|c|c|c|}
\hline Tondage à la main & Table à tondre mécanique & Tondeuse hélicoïdale \\
\hline $\begin{array}{l}\text { Matériels et location } \\
\text { de l'atelier : } 8552 \mathrm{fr} \text {. } \\
\text { (20 tables et } 40 \text { forces) }\end{array}$ & $\begin{array}{l}\text { Matériel et location } \\
\text { de l'atelier : } 25000 \mathrm{fr} \text {. } \\
\text { ( } 40 \text { tables et } 40 \text { forces avec } \\
\text { mouvements et accessoires) }\end{array}$ & $\begin{array}{l}\text { Une tondeuse et location } \\
\text { de l'atelier : } 20000 \mathrm{fr} \text {. }\end{array}$ \\
\hline $\begin{array}{l}\text { Main-d'œuvre : } 32376 \text { fr. } \\
\text { ( } 40 \text { tondeurs à } 2 \text { fr } 50 \\
\text { pour } 300 \text { jours, emou- } \\
\text { lage et renouvellement } \\
\text { des forces) }\end{array}$ & $\begin{array}{l}\text { Main-d'œuvre : } 17106 \mathrm{fr} \text {. } \\
\text { (15 tondeurs - } 1 \text { pour } \\
3 \text { tables - émoulage } \\
\text { des forces, entretien, force } \\
\text { motrice) }\end{array}$ & $\begin{array}{l}\text { Main-d'œuvre : } 3500 \mathrm{fr} \text {. } \\
\text { ( } 1 \text { tondeur à } 3 \mathrm{fr} \text { pour } \\
300 \text { jours et deux femmes } \\
\text { à } 1 \mathrm{fr} 50 \text { pour } 300 \text { jours, } \\
\text { entretien, force motrice) }\end{array}$ \\
\hline
\end{tabular}

Source : Notice sur une nouvelle machine à tondre les draps appelée tondeuse ou Forces Hélicoïdes, Vve Courcier, Paris (s. d.).

De nombreux modèles de machines sont proposés entre 1800 et 1830 pour automatiser l'opération complexe et coûteuse du tondage des draps. Les simples " tables à tondre " se contentent d'abord de mécaniser le maniement des anciennes forces en utilisant un moteur extérieur (Fig. 1), alors que les tondeuses dites rotatives, ou " hélicoïdales ", également appelées au début du XIX $\mathrm{XX}^{\mathrm{e}}$ siècle "forces continues ", "parfait tondeur " ou " grande tondeuse ", introduisent une trajectoire inédite après 1815 (Fig. 2). Au lieu de tondre dans le sens de la largeur avec des forces, comme le faisait l'artisan, cette machine tond en longueur au moyen de lames tranchantes qui sont adaptées à une roue ou à un cylindre tournant. L'ancien mouvement longitudinal des forces est supplanté par un "mouvement circulaire continu ", ce qui permet une accélération considérable de la productivité, perçue comme une menace pour la main-d'œuvre ${ }^{19}$. Plusieurs modèles

18. Gayot, 2002, p. 30 ; Jarrige, 2012 ; Mouchel, 1885 ; Pilisi, 1954-1956.

19. Les divers modèles de cette mécanique ont fasciné les technologues du XIX ${ }^{e}$ siècle qui se sont attachés à décrire par le menu leur fonctionnement, voir notamment : Borgnis, 1820, t. VII : " Des machines qui servent à confectionner les étoffes ", " Tondage ", p. 310-330. 


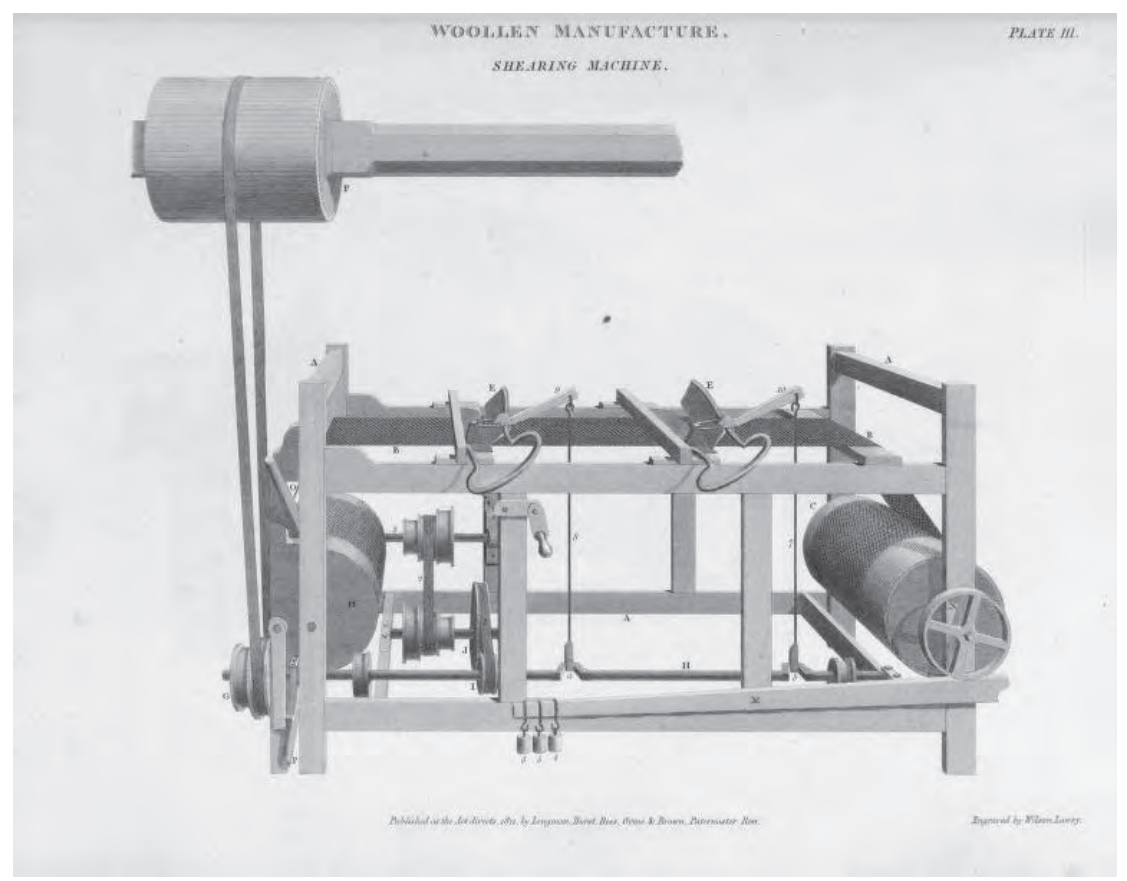

\section{Fig. 1. - Table à tondre mécanisée ou forces mécaniques (Shearing Machine)}

Abraham Rees, The Cyclopaedia; or, Universal Dictionary of Arts, Sciences, and Literature, Plates, vol. IV, Londres, Longman, Hurst, Rees, Orme \& Brown, 1820, « Wollen Manufacture », planche III.

\section{Source : Internet Archive, University of Toronto - Robarts Library, https://archive.org/details/cyclopaediaplates04rees/page/n359/ mode/2up?q=plate (consulté le 23 octobre 2020).}

de machines voient le jour comme celle de l'anglais Price qui prend une patente en 1815, elle est ensuite adaptée lorsqu'elle est importée sur le continent par divers constructeurs mécaniciens.

Les constructeurs déploient une intense activité pour intéresser le public et les autorités à cette machine. Ils ouvrent des ateliers publics pour convaincre les fabricants et la main-d'œuvre, ils mettent sur pied des stratégies publicitaires et éditent une brochure qui vante les mérites de la nouvelle méthode qui « tond parfaitement les draps les plus fins, ainsi que les draps ordinaires, les casimirs, les cachemires et les étoffes appelées Mérinos ; on garantit la Tonte égale à ce que les autres procédés peuvent 
faire de mieux pour le fini, et bien supérieure pour la régularitée ${ }^{20}$ ». Selon les calculs présentés en annexe de cette brochure (tableau ci-dessus) la tondeuse permettrait de diviser le coût de la main-d'œuvre par près de dix pour un investissement seulement 2,5 fois supérieur à celui du tondage à la main. Les fabricants promettent ainsi que les fiers tondeurs, souvent portés à l'insubordination, pourront être remplacés par « deux jeunes garçons ou deux femmes " pour conduire le drap. Ces mécaniques sont construites par des fabricants comme Cockerill ou Ternaux alors qu'un véritable marché européen voit le jour, mais elles provoquent des réactions très variables et parfois violentes dans les places de fabriques européennes.

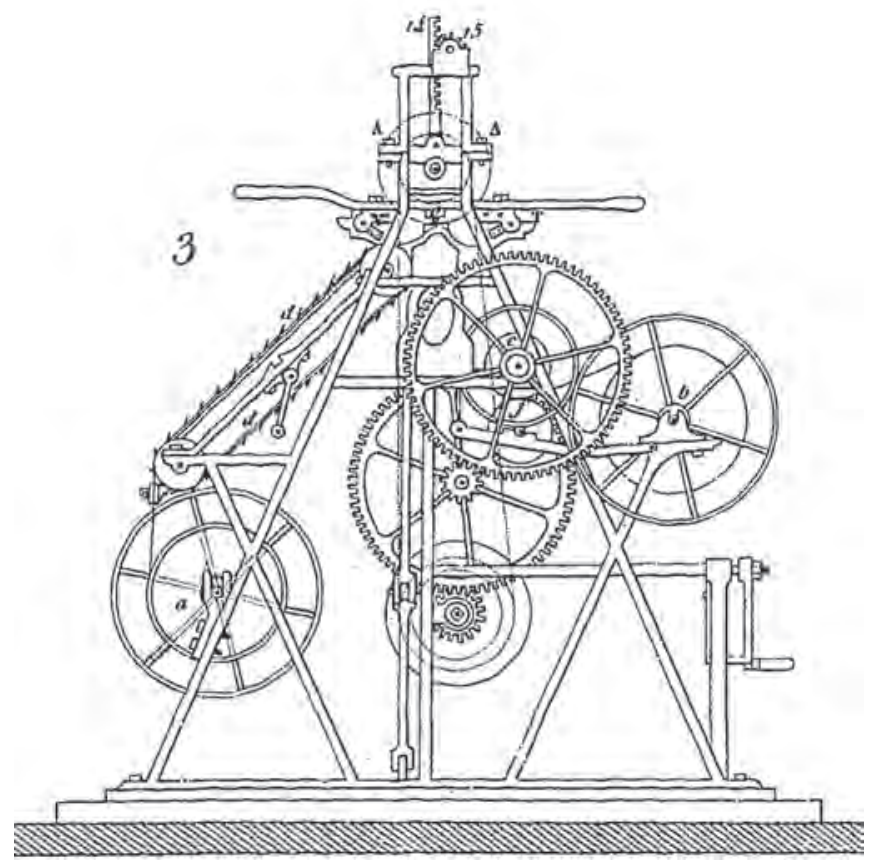

\section{Fig. 2. - Tondeur à lames hélicoïdes de M. Stephen Price}

Giuseppe Antonio Borgnis, Traité complet de mécanique appliquée aux arts, t. vII. Des machines qui servent à confectionner les étoffes, Paris, Bachelier, 1820, pl. 43, détail.

Source : gallica.bnf.fr / Bibliothèque nationale de France, https://gallica.bnf.fr/ark:/12148/bpt6k110288t/f388

20. Notice (s. d.). 
Les violences les plus graves ont lieu comme on l'a dit en Angleterre. Les premières laineuses mécaniques suscitaient déjà des plaintes récurrentes au XVIII ${ }^{\mathrm{e}}$ siècle, qui atteignent leur apogée lors des troubles du Wiltshire de 1802. Les travailleurs et les petits maîtres parviennent à freiner leur diffusion jusqu'au début du XIX ${ }^{\text {e }}$ siècle, en arguant notamment du fait que les pièces de draps lainées mécaniquement n'auraient pas été bien tondues ${ }^{21}$. Ce sont ensuite les tables à tondre mécaniques qui provoquent troubles et désordres. En mars 1811, un voyageur français visitant Leeds souligne que les tondeurs "voient de mauvais oil l'introduction récente d'une machine mise en mouvement par la pompe à feu, qui menace de les supplanter ; ils murmurent hautement, et l'on craint qu'il n'y ait du désordre ${ }^{22}$ ". L'hostilité de la main-d'œuvre atteint son apogée lors des troubles du luddisme en 1811-1812 au cours desquels de nombreuses mécaniques sont mises hors service. L'arrivée de ces machines sur le continent européen provoque des réactions très variables. Dans l'Europe méridionale ou dans la France du Nord, aucun conflit n'est signalé. La répression des émeutes antérieures, le manque chronique de main-d'œuvre dans la draperie normande, comme la concurrence du coton, expliquent en partie l'absence de troubles.

En revanche, l'arrivée des tondeuses provoque des désordres fréquents dans les villes du Midi exclusivement vouées à la production des draps ou dans certaines places lainières de Belgique et de l'espace germanique. À Vienne, dans le Dauphiné, les désordres contre la " Grande Tondeuse » éclatent en février 1819, provoquant l'inquiétude des autorités et des fabricants. Dans une pétition qu'ils adressent au maire de la ville, huit maîtres tondeurs dénoncent « la mécanique plus pernicieuse qu’utile nommée la grande tondeuse " qui annonce, selon eux, "la suppression générale des bras ». Dans les années qui suivent, des troubles éclatent à Lodève, Clermont-L'Hérault, Bédarieux, Castres, Carcassonne ou Limoux, autant de petites places lainières entrées en crise ${ }^{23}$. De nombreux facteurs expliquent cette conflictualité : d'abord la conjoncture de crise et de déclin, après une phase de forte croissance, qui rend ces centres textiles méridionaux particulièrement vulnérables. Ensuite, l'organisation du travail elle-même qui, contrairement à

21. Randall, 1991, p. 110.

22. Simond, 1816, t. 2, p. 103-104.

23. Manuel, 1938 ; Johnson, 1995 ; Jarrige, 2009, p. 76-82. 
Sedan où l'étape du tondage est concentrée précocement, voit le maintien de petits ateliers composés de quelques ouvriers.

Dans les territoires germaniques, eux-mêmes très divers et organisés depuis longtemps sur un mode proto-industriel ${ }^{24}$, les réponses à l'arrivée des tondeuses rotatives au cours des années 1820 sont également ambivalentes. En Saxe, l'accumulation des capitaux est suffisamment importante et précoce pour permettre à certains grands Verleger comme Adolf Gottlieb Fiedler, propriétaire d'une fabrique près d'Oederan, d'introduire dès 1818 des machines à tondre les draps produites par le baron de Neuflize ${ }^{25}$. Dans l'ensemble, pourtant, les fabricants hésitent. En Saxe, aucune autre manufacture de draps ne semble avoir adopté la tondeuse diffusée par le fabricant Cochelet en 1821, les fabricants continuent à se servir des rudimentaires "forces de tondeur mécanisées », construites à Berlin par Cockerill, qui sont pourtant loin d'avoir le rendement et les avantages de la tondeuse circulaire Cochelet. À Brünn, en Moravie, dans l'Empire d'Autriche, un conflit éclate d'ailleurs en 1819. Comme en France au même moment, l'arrivée de la tondeuse circulaire provoque l'effervescence dans le milieu des maîtres tondeurs et de leurs compagnons. Après que le quotidien de Vienne Der Osterreichische Beobachter ait rapporté dans son numéro du 18 mars 1819 les évènements de Vienne (Isère), au cours desquels la ville se soulève, les tondeurs tentent à leur tour de s'insurger, mais l'émeute tourne court $^{26}$. Un autre fabricant du comté de Berg échoue à introduire une mécanique à cause du refus de la main-d'œuvre. Il semble que les Verleger aient choisi d'adopter les tables à tondre plutôt que les procédés les plus récents pour éviter de susciter la colère de leurs ouvriers.

En Belgique, Laurent-François Déthier prévoit les conséquences les plus funestes à la suite de l'arrivée de ces machines : «Et à quoi ne doit-on pas s'attendre des suites infaillibles et trop funestes de l'introduction générale de ces mécaniques, non seulement dans le département de l'Ourthe mais encore dans ceux de la Roer et de la Meuse inférieure que vivifient aussi les célèbres manufactures de draps d'Aix la Chapelle, de Borcheid, de Vals, Monjoie, etc.... ${ }^{27}$. " Au début du XIX ${ }^{\mathrm{e}}$ siècle, Verviers s'affirme comme le

24. Kisch, 1980.

25. Gayot, 2003.

26. Pilisi, 1957.

27. Delrée et Hélin, 1986-1987. 
premier centre continental de la draperie cardée, devant Sedan et Elbeuf ${ }^{28}$. L'arrivée des tondeuses rotatives y provoque également des troubles en janvier 1819, lorsque des « billets de provocation à la révolte [...] distribués et répandus dans la ville » invitent les ouvriers à "détruire une machine à tondre les draps nouvellement mise en activité29 ». Dans un contexte de crise, la condition des travailleurs s'est dégradée dans la région verviétoise. À la Reid par exemple, les autorités locales notent que les « habitants sont plongés dans l'indigence " depuis que les " mécaniques " se sont répandues $^{30}$. De nouveaux troubles renaissent dix ans plus tard à l'occasion de l'annonce de la Révolution bruxelloise de 1830. Le 28 août 1830, la foule arborant le drapeau français se rend à l'hôtel de ville aux cris de "Vive Napoléon ! À bas Guillaume ! Brisons les machines !». Elle s'empare des armes de la Garde communale et saccage plusieurs maisons. Les autorités ne parviennent à restaurer le calme qu'en promettant une baisse du prix du pain et la mise hors service des machines à tondre ${ }^{31}$. Le 30 août au matin, plusieurs fabricants font démonter leurs tondeuses et les exposent à la vue de la population qui s'en empare et les promène sur des charrettes ${ }^{32}$. Chez le fabricant Coumont-Grosfils, « un attroupement populaire [...] a enlevé, brisé et détérioré les machines à tondre servant à sa fabrique de draps ${ }^{33}$ ».

L'histoire des machines à tondre les draps en Europe montre toute la complexité des relations entre travail, mécanisation et savoir-faire professionnels autour de 1830. Les hésitations de nombreux fabricants s'expliquent par de multiples facteurs : le prix élevé, la productivité de la tondeuse, son rendement formidable. Mais celui-ci peut paradoxalement se retourner contre elle car les petits fabricants, qui n'ont pas assez de pièces à faire tondre, hésitent devant une telle dépense, n'ayant du travail pour un procédé si efficace que deux à trois jours par semaine. Dans de nombreux endroits, le débit demeurait trop limité pour justifier l'acquisition d'une

28. Lebrun, 1948 ; Gayot, 2002.

29. AEL, Fonds hollandais, 803 : rapports périodiques de police de l'arrondissement de Verviers, janvier 1819.

30. AEL, Fonds hollandais, 3539 : états des fabriques et ateliers à Verviers au 31 décembre 1819 ; Fonds hollandais, 3521 : commune de La Reid (canton de Spa).

31. Le 29 août, la Commission de Sûreté publique a dû prendre un arrêté défendant l'emploi des tondeuses, il est abrogé dès le 8 septembre [Fohal, 1930, p. 20-21 et 68].

32. Lejaer, 1906, p. 14; Fohal, 1930, p. 21.

33. Arch. Communales de Verviers, Farde 39 : Indemnisation Coumont-Grosfils, réclamation $\mathrm{n}^{\circ} 16$. 
telle machine. L'effet de ces machines fut donc ambigu : dans de nombreux espaces, l'augmentation de la production fut assez forte pour que les emplois perdus ne soient finalement pas très nombreux. Loin de remplacer les anciennes méthodes de tondage, les tondeuses hélicoïdales s'ajoutent à elles pour la réalisation des premières tontes ou pour certains types de produits spécifiques. La mécanisation a néanmoins comme effet d'accentuer la différenciation au sein du monde du travail chargé des apprêts : à côté des anciens tondeurs à la main, une foule d'hommes nouveaux, de femmes et d'enfants, pénètrent dans les ateliers, selon des rythmes variables et fluctuants. Sur la longue durée, le principal résultat de cette mécanisation du tondage des draps fut de laminer l'ancien contrôle exercé par les travailleurs sur le processus de production et le rythme du travail. Le travail qualifié réalisé par des équipes de deux tondeurs était désormais exécuté par un homme et un enfant, parfois le fils du tondeur.

\section{Les machines en débat}

Dès les années 1820, l'économiste Jean-Baptiste Say et le philosophe Saint-Simon font de l'industrie une force pacificatrice qui doit refermer la Révolution française et son cycle de violences en substituant « le gouvernement des choses à celui des hommes ». En favorisant l'appropriation des ressources de la nature, l'industrie doit éclipser la misère interprétée comme l'héritage du passé aristocratique. Elle devient aussi la source et le symbole de l'ascendant que l'Europe acquiert sur le reste du monde. L'industrialisation - même si le mot lui-même ne se répand qu'autour de 1900 - devient ainsi une nouvelle source de confiance dans l'avenir, à partir duquel seront interprétées l'évolution des sociétés et leurs relations mutuelles, selon une échelle distinguant les zones avancées et celles en retard. Dans la première moitié du XIx ${ }^{e}$ siècle, dans certains endroits comme Manchester dans le Lancashire (Angleterre) ou Lowell en NouvelleAngleterre (États-Unis) de vastes usines textiles mécanisées et concentrées naissent en employant une main-d'œuvre souvent peu qualifiée, issue des campagnes, produisant en masse des produits de plus en plus standardisés. Ces usines ont beaucoup retenu l'attention et frappé les observateurs du XIX ${ }^{e}$ siècle. Dans les grandes régions cotonnières d'Europe, les filature - symboles de ces premières usines modernes recourant aux vastes machines - accueillent déjà plusieurs centaines d'ouvriers en moyenne dès 
les années 1830 (près de 300 en Alsace ou dans le Lancashire vers 1840); dès 1816 deux usines de Manchester dépassent déjà le millier d'ouvriers.

Mais, au-delà des discours élogieux et de ces situations exceptionnelles, l'industrialisation demeure fragile et emprunte à ses débuts des chemins variés. Avant 1850, elle demeure d'ailleurs limitée, et les spécialistes relativisent souvent son impact en soulignant combien - même en GrandeBretagne - la croissance du PIB est faible (pas plus de 1,5\%) alors que le poids des activités agricoles et artisanales demeure essentiel. Les ateliers artisanaux urbains et les micro-établissements insérés dans le tissu rural, qui prolongent les dynamiques proto-industrielles antérieures, demeurent la forme la plus fréquente de production industrielle en Europe au milieu du XIX ${ }^{e}$ siècle. Ce qu'on appelle le modèle de "Birmingham ", fondé sur la sous-traitance et le semi de petites entreprises travaillant de façon artisanale, se retrouve dans la petite quincaillerie de Saint-Étienne et SaintChamond (France), comme dans la coutellerie de Sheffield et Solingen (Allemagne).

Dans la première moitié du $\mathrm{XIX}^{\mathrm{e}}$ siècle, les plaintes ouvrières et doutes à l'égard des machines ne sont pas rares et mobilisent souvent les mêmes arguments. Des fileurs de coton de Paris rejettent ainsi les mécaniques anglaises " qui coupent les bras à tous les ouvriers". Ce type de formule a la vie dure et cela dans des groupes très différents. Après la révolution de février 1848, les coupeuses de poils de lapin se plaignent à leur tour de la multiplication des mécaniques, "ce qui coupe les bras aux ouvrières ". L'invocation récurrente des " bras " renvoie à la perception singulière des formes de la propriété : pour les ouvriers, les bras forment la propriété de base des individus, ils symbolisent à la fois le travail incorporé et les compétences acquises au terme d'un long apprentissage. Alors que les outils du métier étaient le prolongement du corps, les machines de l'ère industrielle deviennent au contraire des éléments d'étrangeté inorganique ${ }^{34}$. À Paris, les imprimeurs typographes - symboles des gens de métiers urbains et qualifiés - s'en prennent aux presses mécaniques récemment importées d'Angleterre qu'ils détruisent dans la foulée des Trois Glorieuses de juillet 1830. Eux aussi voient dans les nouvelles mécaniques " des rivales qui viennent nous casser les bras ». Protestant contre la mise en place d'une édition à deux vitesses, où les ouvrages de luxe restent produits artisanalement, là

34. Jarrige, 2009. 
où les grands tirages sont réalisés mécaniquement par des ouvriers souspayés. Certaines communautés paysannes se mobilisent également contre la mécanisation du battage des grains, opération décisive qui offrait du travail aux ouvriers agricoles : l'Angleterre connaît dès 1830 de violentes émeutes dites du "Capitaine Swing"35 ", en France en 1847 des batteuses mécaniques sont également détruites, bien souvent à la faveur de troubles frumentaires.

Ces désordres viennent du fait que les mutations techniques recomposent les mondes du travail. Elles sont accusées d'accentuer l'exploitation et la prolétarisation qui s'incarnent notamment dans le travail des enfants de plus en en plus condamné et repoussé, alors que la mécanisation multiplie les tâches où ils peuvent être employés, à l'image des rattacheurs dans les filatures textiles mécanisées. Chez des auteurs comme Balzac, Hugo, Vigny la découverte des grandes machines productives accompagne une « mélancolie industrielle » qui irrigue largement les écrits du temps. On la trouve aussi chez l'historien français Jules Michelet, qui observe le gigantisme de l'industrie britannique lors d'un voyage outre-Manche en 1834. Dans les années 1840 , il invente d'ailleurs le mot " machinisme " auquel il donne une signification morale puisqu'il l'utilise pour condamner le système industriel à l'anglaise et les nouveaux rapports sociaux qu'il produit. Dans Le Peuple (1846), il consacre tout un chapitre à décrire la "servitude de l'ouvrier dépendant des machines ". Impressionné par les "êtres d'acier " qui asservissent de plus en plus "l'être de sang et de chair ", il affirme néanmoins que l'on continuera de préférer aux « fabrications uniformes des machines les produits variés qui portent l'empreinte de la personnalité humaine ${ }^{36}$ ». Mais si la machine est indéniablement un " puissant agent du progrès démocratique » en "mettant à la portée des plus pauvres une foule d'objets d'utilité ", elle a aussi son revers terrible : elle crée un "misérable petit peuple d'hommes-machines qui vivent à moitié [et] qui n'engendrent que pour la mort ". Chez Michelet, la dénonciation du machinisme passe par la condamnation de ses effets sur les ouvriers, victimes d'ennui et d'abrutissement :

Le cœur bat-il dans cette foule ? bien peu, son action est comme suspendue; il semble, pendant ces longues heures, qu'un

35. Griffin, 2012.

36. Michelet, 1974 [1846], p. 98 ; Viallaneix, 1979 ; Jarrige, 2017. 
autre cœur, commun à tous, ait pris la place, cœur métallique, indifférent, impitoyable, et que ce grand bruit assourdissant dans sa régularité, n'en soit que le battement.

Le nouveau système des fabriques mécanisées est accusé de transformer peu à peu l'homme en machine sans âme, à mille lieues de l'ancien artisan travaillant dans un atelier ou à domicile : "Le travail solitaire du tisserand était bien moins pénible. Pourquoi ? C'est qu'il pouvait rêver. La machine ne comporte aucune rêverie, nulle distraction » affirme encore Michelet ${ }^{37}$. Tout en laissant entrevoir une possible réappropriation des machines dans la future société communiste, Karl Marx et Friedrich Engels observent également, en 1848, qu'avec l'industrie moderne «l'ouvrier devient un simple accessoire de la machine » et que les masses prolétaires sont « les esclaves de la machine ${ }^{38}$ ". Chez Marx la définition du prolétariat repose sur trois piliers essentiels : il est d'abord le produit de l'organisation industrielle du travail et il n'existe que dans et par la grande industrie ; mais le prolétariat est aussi le producteur de la plus-value qui permet la reproduction élargie du capital, et à ce titre il est la source de tout le développement économique et technologique ; enfin la condition prolétarienne se caractérise par l'insécurité fondamentale. Cette insécurité n'est pas liée aux seuls aléas de la conjoncture, mais au mode de production capitaliste lui-même qui a besoin de l'existence d'une armée de réserve industrielle pour fonctionner. Les prolétaires sont donc :

La classe des ouvriers modernes qui ne vivent qu’à la condition de trouver du travail et qui n'en trouvent que si leur travail accrôit le capital. Ces ouvriers, contraints de se vendre au jour le jour, sont une marchandise au même titre que tout autre article de commerce; ils sont exposés, par conséquent, de la même façon à toutes les vicissitudes de la concurrence, à toutes les fluctuations du marché. Le développement du machinisme et la division du travail, en faisant perdre au travail de l'ouvrier tout caractère d'autonomie, lui ont fait perdre tout attrait ${ }^{39}$.

37. Michelet, 1974 [1846], p. 98-99.

38. Engels et Marx, 1998, p. 31-32.

39. Ibid. 


\section{Civiliser les machines : nouvelles qualifications et travail émancipé ?}

À côté des craintes, le xix ${ }^{\mathrm{e}}$ siècle voit surtout la prolifération des tentatives pour reconquérir et civiliser le monde mécanique en imaginant l'ouvrier en technicien, et la machine en auxiliaire capable de suppléer le corps fini et fragile du travailleur. Le développement technique suscite en effet l'apparition de nouvelles professions, de nouveaux spécialistes, mécaniciens, fabricants de machines, chauffeur, contremaîtres, sans parler des ingénieurs spécialisés dans la maîtrise de ce nouveau monde technique... Chez les technologues et ingénieurs, les machines deviennent d'ailleurs de nouveaux esclaves d'aciers qui doivent émanciper le travailleur en le libérant des tâches éprouvantes. Pour désamorcer les inquiétudes, et pacifier la relation des hommes aux machines de plus en plus dangereuses au quotidien et à l'origine de nombreux accidents, une nouvelle représentation des machines comme prolongement du corps ouvrier se met ainsi en place. Les machines deviennent des quasi-personne dotées de pouvoirs magiques. Plusieurs historiens ont récemment examiné comment la machine est devenue un fétiche dans l'Angleterre du $\mathrm{XIX}^{\mathrm{e}}$ siècle, une source d'illusions et de myopie sur le monde, nourrie par l'idéologie de la modernité technologique, mais fondée sur de multiples formes de prédations bien réelles à l'égard des milieux naturels et des humains ${ }^{40}$. Dans les écrits du chimiste anglais Andrew Ure par exemple, le métier à filer automatique est conçu comme une quasi-personne, il est doté de l'élan vital et devient un Iron Man. Le même type de formule revient sous la plume de Michelet au début de son histoire inachevée du XIx ${ }^{\mathrm{e}}$ siècle lorsqu'il évoque la manufacture de Watt et Boulton "produisant sans mesure ses ouvriers de fer, de cuivre, par lesquels l'Angleterre eut bientôt la force de quatre cents millions d'hommes ${ }^{41}$ ". Une autre métaphore, plus fréquente encore, fait des machines et des outils de nouveaux esclaves chargés d'assister les hommes et de palier à la faiblesse de leur corps. Lors de l'Exposition universelle de 1862 à Londres, l'économiste et ancien saint-simonien Michel Chevalier fait ainsi de la machine le prolongement du corps : « les outils sont pour l'homme des organes supplémentaires par lesquels il

40. Hornborg, 2001 ; Malm, 2016 ; Macleod, 2007.

41. Michelet, 1893-1898, vol. 24 : "Histoire du XIx siècle », Préface, p. 10 ; Ure, 1835, p. 367. 
peut aborder une infinité d'opérations». Loin d'abrutir le corps et l'esprit comme certains le craignent, les machines doivent assister et accroître ses capacités, rendant possible son émancipation là où l'idée de prolétarisation insistait sur l'abolition de toute autonomie et de toute libertét ${ }^{42}$.

Tout au long du $\mathrm{XIx}^{\mathrm{e}}$ siècle, la question des machines demeure l'enjeu d'un vaste débat sur sa possible réappropriation. Comment transformer la machine qui menace d'absorber l'homme en instrument de son émancipation ? Le projet des réformateurs sociaux qui inventent la "science sociale " après 1830 propose d'imaginer une technologie qui favoriserait l'autonomie et accompagnerait la réalisation de l'humanité présente en chacun. Pour les penseurs dits socialistes, l'avenir doit ainsi permettre l'avènement d'un ouvrier émancipé, façonner un pur technicien compétent qui se consacrera aux tâches intellectuelles, laissant les activités machinales aux artefacts, inaugurant ainsi une autre vision de l'innovation technologique. Loin de rejeter le monde mécanique, il s'agit de tenter de le reconquérir en définissant les caractéristiques d'une " machine romantique » - alternative à celle des ingénieurs et des économistes - capable de réenchanter le monde et la sphère du travail en produisant un ordre plus démocratique ${ }^{43}$. Ce thème se retrouve sous des formes variables chez des penseurs radicaux comme le britannique Robert Owen ou les français Charles Fourier, Étienne Cabet ou encore Pierre J. Proudhon. Chez Fourier, c'est la distribution passionnelle des travaux qui doit éviter le surgissement de l'ouvrier machine rivé à une seule tâche aliénante. Grâce à sa " théorie du travail attrayant ", Fourier imagine d'accroître les rendements et l'efficacité des travailleurs sans recourir à la parcellisation déshumanisante des tâches ou aux machines, et en assurant l'assouvissement des plaisirs et passions de chacun $^{44}$. Ses disciples retraduiront le langage fouriériste en l'adaptant au cadre de la grande usine, comme dans ce texte de propagande décrivant en détail le quotidien d'un phalanstère et rédigé peu avant la révolution de février 1848 par l'ouvrier teinturier Mathieu Briancourt :

Ici, comme partout, les machines font à peu près toute la portion fatigante de la besogne : la machine fournit la force, l'homme

42. Michel Chevalier (dir.), Exposition universelle de Londres de 1862, cité dans Riot-Sarcey, 2016, p. 207.

43. Tresch, 2012 ; Jarrige, 2016.

44. Beecher, 1990. 
l'intelligence et sa tâche se borne, dans l'industrie manufacturière, à surveiller, à diriger, à ajuster. Vous ne devez donc pas être surpris de voir plusieurs dames enrôlées parmi nos tourneurs et nos scieurs de long, dont les métiers si pénibles autrefois étaient abandonnés aux esclaves. Si vous voyez divers systèmes de machines appliqués à des opérations identiques, c'est afin d'entretenir l'émulation parmi les travailleurs dont chaque groupe donne la préférence à une machine différente de celles dont se servent les groupes rivaux ${ }^{45}$.

Chez le communiste Étienne Cabet, grâce au progrès poussé des machines les ouvriers peuvent également devenir " inventeurs " et " directeurs " de machines. Dans la République icarienne décrite dans Le Voyage en Icarie (1840), c'est en effet la collectivité qui est propriétaire des moyens de production. Loin de supprimer l'artisan indépendant, Cabet affirme que c'est la machine qui permet de restaurer l'autonomie artisanale en faisant des ouvriers des « directeurs » de machines. En abolissant la propriété privée, le système communiste doit détruire les relations hiérarchiques incarnées par la figure de l'ingénieur, et reconstituer l'autonomie de l'artisan menacée par la concentration croissante du capital. La force du discours icarien et son efficacité propagandiste viennent de sa capacité à animer la machine en la dotant d'attributs quasi magiques tout aux bénéfices des classes populaires et ouvrières. Mais c'est sans doute Proudhon qui a été le plus loin dans le renversement dialectique du thème de l'ouvrier aliéné par les machines. Là où beaucoup de ses contemporains voyaient dans la division du travail une conséquence inéluctable du machinisme, Proudhon insiste au contraire sur l'ambivalence et la complexité du processus. Pour lui, le machinisme permet en effet de rompre avec les effets délétères d'une division excessive du travail en requalifiant le travailleur :

L'apparition incessante des machines est l'antithèse, la formule inverse de la division du travail ; c'est la protestation du génie industriel contre le travail parcellaire et homicide. Qu'est-ce en effet qu'une machine ? Une manière de réunir diverses particules de travail que la division avait séparée. Toute machine peut être définie : un résumé de plusieurs opérations, une simplification de ressorts, une condensation du travail, une réduction de frais ${ }^{46}$.

45. Briancourt, 1848, p. 127-128.

46. Proudhon, 1846, chap. IV : «Des machines ». 


\section{Corps à corps quotidien avec la machine}

Mais au-delà des discours à visée théorique et englobante, c'est évidemment l'hétérogénéité des situations qui domine alors que l'impact des machines sur les mondes du travail, ses qualifications, sa structuration interne, varie considérablement selon les métiers et les régions d'Europe. C'est en Angleterre, puis en Belgique et, plus tard, dans l'espace germanique que se créent les premières grandes concentrations industrielles fondées sur le textile, la sidérurgie et le charbon ; c'est là qu'une partie, parfois majoritaire, de la population - artisans et compagnons déclassés, mais surtout paysans déracinés - va progressivement constituer cette immense classe n’ayant pour vivre que sa simple force de travail, laquelle ne lui apporte ni reconnaissance, ni sécurité matérielle. Le langage du « prolétariat » s'étend dans ce contexte pour désigner celui qui voit son savoir-faire concurrencé par les nouvelles logiques techniques et organisationnelles du travail industriel, et son mode de vie détruit par le déracinement dans les grandes villes et les usines. Pour simplifier, on peut d'ailleurs considérer que c'est la machine qui définit le prolétariat dans les discours du temps. Étant donné la faiblesse de son salaire, le travailleur est en effet incapable d'acquérir des moyens de production que le développement industriel rend toujours plus onéreux, imposant la concentration croissante de capitaux énormes. L'expérience du prolétaire se caractérise par une dépossession et un contrôle social renforcé. L'industrialisation du XIX ${ }^{\mathrm{e}}$ siècle voit en effet surgir une exigence croissante de discipline et de contrôle de la main-d'œuvre et de son activité.

Dans les discours des fabricants, ingénieurs et économistes, le projet de mécanisation industrielle s'énonce fréquemment via le langage du travail discipliné et moralisé et une science du travail rationalisé voit peu à peu le jour. Claude Anthelme Costaz - conseiller d'État, préfet, membre du Tribunat, directeur général des Ponts et Chaussées, l'un des personnages clés de l'appareil économique d'État sous le Directoire et l'Empire - expliquait déjà en 1815 que "la découverte des machines rend aujourd'hui impuissante la mauvaise volonté des ouvriers puisqu'ils ne sont plus, comme autrefois, des instruments indispensables à l'activité des manufactures et qu'on peut, sans inconvénient, les remplacer par des hommes nouveaux et manquant d'expérience ${ }^{47}$ ".

47. Costaz, 1818, p. 149. 
L'exemple du travail des tisserands illustre ces transformations radicales en Europe et a souvent été décrit. Encore assez rares avant 1880 en dehors des îles Britanniques, les vastes tissages mécaniques automatisés se généralisent à la fin du siècle en provoquant d'abondants débats. Après les métiers à domicile perfectionnés, puis le métier mécanique qui tisse seul, actionné par une force extérieure, mais pour lequel l'ouvrier doit encore changer les canettes, vient le temps des machines automatiques que l'ouvrier n'a plus qu'à surveiller. C'est le cas du métier dit Northrop mis au point par James A. Northrop, un technicien d'origine britannique installé aux États-Unis. D'abord expérimentée en 1893 lors de l'Exposition universelle de Chicago, la machine est commercialisée dès l'année suivante alors que l'Amérique du Nord devient une grande puissance industrielle et un modèle en Europe. 30000 exemplaires sont installés aux ÉtatsUnis entre 1895 et 1900 . Ils permettent une spectaculaire économie de main-d'œuvre puisqu'un ouvrier peut désormais conduire jusqu'à 16 voire 24 métiers à la fois. Ils pouvaient par ailleurs tisser des articles variés et dès 1896 plus de 8000 mécaniques de ce type sont en service dans 38 tissages de Nouvelle-Angleterre ${ }^{48}$. La rapidité de cette adoption s'explique par la forte croissance industrielle que connaissent alors les États-Unis où le manque de main-d'œuvre favorise l'adoption des métiers économisant les bras. En Europe en revanche, leur diffusion est bien plus lente et provoque de nombreux doutes là où le tissage existait depuis longtemps et avait soudé d'importantes communautés professionnelles, avec leur culture collective et leur tradition de lutte.

Si les grandes machines productives accentuent sans nul doute la déqualification et la concentration de la main-d'œuvre dans des usines, d'autres comme la machine à coudre créent un mouvement inverse en redynamisant le travail à domicile. La mécanisation ne cesse de remodeler les statuts sociaux et la répartition sexuée des tâches au XIX ${ }^{\mathrm{e}}$ siècle. Là encore, il n'existe pas d'effets univoques et simples même si globalement les femmes se voient reléguer dans les tâches auxiliaires, les travaux d'exécution subordonnés alors que les hommes - mieux payés - contrôlent les postes de commandement, d'encadrement et les machines complexes. Si la mécanisation permet bien une sortie du foyer et l'entrée des femmes sur le marché du travail, l'historienne Michelle Perrot observait que cela n'impliquait

48. Feller, 1966 ; Benoit, 2009. 


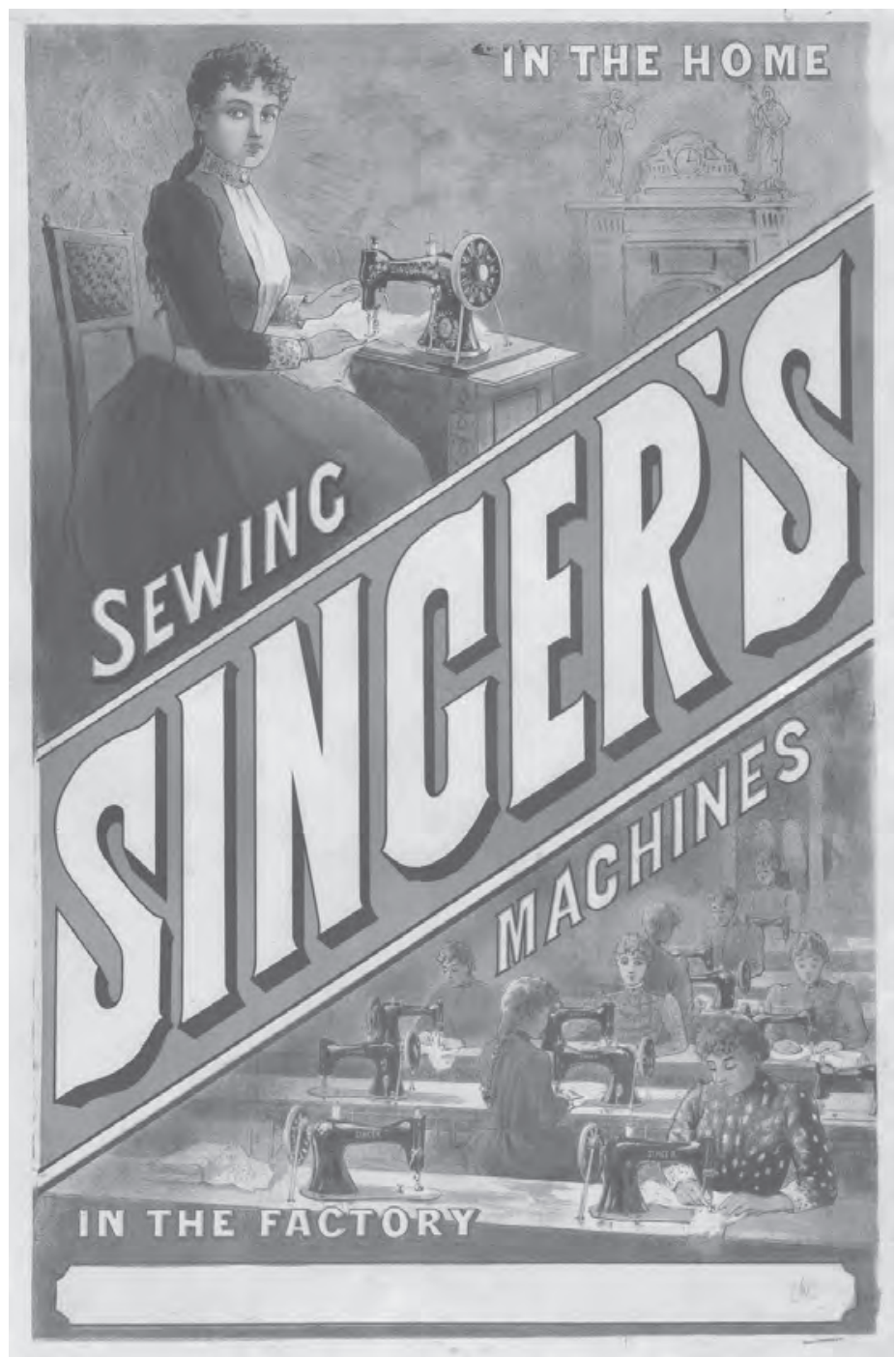

Fig. 3. - Singer's Sewing machines, in the home, in the factory. Affiche Singer (1892)

Source : gallica.bnf.fr / Bibliothèque nationale de France, https://gallica.bnf.fr/ark:/12148/btv1b531276034

" ni leur libération, ni leur promotion, ni leur accès à la technique ${ }^{49}$ ". La machine à coudre, cet « instrument hybride entre ustensiles ménagers

49. Perrot, 1998, p. 182. 
et matériel de production, à la frontière de l'économie domestique et de l'économie industrielle, de la consommation et de la production » offre un bon exemple de ces transformations ${ }^{50}$. Cette " mécanique à faire les coutures $^{51}$ " est à la fois une fabrication industrielle dotée de mécanismes complexes et un objet réalisant des activités domestiques. Ses origines remontent en 1830, mais ce n'est qu'à partir des années 1850, qu'elle commence véritablement à se diffuser en masse à l'échelle internationale, notamment sous l'impulsion de l'entreprise états-unienne Singer ${ }^{52}$. Des milliers de machines de ce type sont rapidement diffusées en Europe, censées reconstituer l'ancien système du travail domestique. Mais dans la pratique ce sweating-system conduit à une paupérisation accrue des femmes dans le cadre du travail en chambre. Loin de restaurer l'ancien travail à domicile la généralisation des machines à coudre prépare l'entrée accrue des femmes dans les usines à l'occasion de la Grande Guerre (Fig. 3).

\section{Nouvelles trajectoires et interrogations fin de siècle}

À la fin du xix ${ }^{\mathrm{e}}$ siècle, le capitalisme industriel et la confiance dans le progrès technique semblent triompher dans le monde. Les opinions contraires subsistent mais elles sont marginalisées et peu audibles. Au sein des nébuleuses réformatrices qui fleurissent en Europe à la fin du XIx ${ }^{\mathrm{e}}$ siècle, le machinisme n'est plus remis en cause comme il l'était au début du siècle, ce sont désormais les conditions sociales de son utilisation qui sont discutées. Après la "grande dépression » des années 1870-1890 vient le temps d'une nouvelle expansion industrielle. Les États-Unis et l'Allemagne s'imposent désormais comme des puissances économiques de premier ordre, tandis que l'industrialisation s'étend à la périphérie de l'Europe (Espagne, Italie, Russie) et, plus lentement, aux mondes coloniaux, qui sont d'abord perçus comme des réservoirs de matière première et de main-d'œuvre à exploiter. Les nouvelles technologies des années 1880-1890, associées à l'intensification des échanges et à l'impérialisme, recomposent le capitalisme comme les formes du travail et accentuent les échanges inégaux à l'échelle globale.

50. Perrot, 1978.

51. Peyrière, 1990.

52. Godley, 2006. 
Le nombre de brevets connaît ainsi une forte croissance, alors que l'alliance de l'électricité, de la chimie et du moteur à explosion recompose le milieu technique. La mécanisation, associée à de nouvelles logiques d'organisation du travail de plus en plus étroitement identifiée au taylorisme, commence à envahir les usines comme les bureaux, en remodelant l'expérience du travail. De nouveaux agencements productifs et techniques voient alors le jour avec le développement de l'industrie des machines-outils, l'interchangeabilité croissante des pièces, ou la production en continu ${ }^{53}$. Visitant l'Exposition universelle de Paris en 1900, symbole de cette nouvelle ère, l'historien américain Henry Adams est par exemple saisi dans la galerie des machines par la vision des grandes dynamos de 12 mètres de haut. Le récit qu'il donne de cette expérience illustre les ambivalences de la fin du XIX ${ }^{e}$ siècle, tiraillé entre espoirs et persistance des doutes à l'égard des grandes technologies industrielles. Loin de célébrer cette grande œuvre de la modernité, il exprime en effet ses craintes devant cette "irruption soudaine de forces entièrement nouvelles ", dont "personne ne semblait $s^{\prime}$ inquiéter ${ }^{54}$ ".

En dépit des inquiétudes qui s'expriment de façon souterraine, la confiance et l'optimisme à l'égard des changements techniques l'emportent. Pour les économistes, les effets néfastes et les problèmes posés localement par le changement technique devront être réglés par l'adaptation de la maind'œuvre, par l'instauration d'un enseignement professionnel et l'intervention croissante des États européens qui commencent à introduire des régulations du travail. L'amélioration de l'hygiène professionnelle ou la sécurisation des systèmes techniques contribuent à l'acclimatation des nouvelles technologies. La "Belle époque " est une période ambiguë à cet égard : les risques et accidents ne cessent de croître, mais leur ampleur est sans cesse atténuée et minimisée. En Europe, les accidents et catastrophes industrielles liés à la fragilité et aux incertitudes technologiques semblent connaître un apogée en termes de nombre de victimes entre les années 1880 et 1920, avant de décliner ensuite. À la fin du XIx e siècle, alors que le suffrage universel et les organisations ouvrières se structurent, des régulations et mesures de protections sont adoptées pour atténuer ces dangers et éviter la montée des contestations. Dans la plupart des pays européens, à la suite notamment des lois allemande et française de 1884 
et 1898, des législations sur les accidents et les risques industriels sont ainsi adoptées pour sécuriser les installations. Elles aboutissent à la socialisation des risques technologiques par les assurances mais aussi à leur acceptation comme un phénomène normal, voire naturel, des sociétés industrielles 55 . Même si ces normes peinent à être appliquées, et s'il existe de multiples résistances patronales et ouvrières, elles atténuent les principaux dangers au travail en imposant un cadre plus strict pour le nettoyage, le graissage ou le débrayage des machines, en contraignant les industriels à s'équiper de dispositifs protecteurs censés résorber les risques antérieurs liés aux dysfonctionnements des engrenages.

La question de l'impact de la mécanisation du travail ressurgit néanmoins lorsqu'apparaît progressivement la catégorie statistique du chômage. Le chômage commence à être pris en compte à la fin du $\mathrm{XIX}^{\mathrm{e}}$ siècle dans les recensements et il donne lieu à une conférence internationale à Paris en $1910^{56}$. Si dans certaines enquêtes officielles, "les transformations rapides des machines » sont mentionnées comme source de chômage, dans l'ensemble l'expansion du machinisme est d'abord vue comme une solution aux crises et à l'instabilité de l'emploi ${ }^{57}$. La théorie économique et les réformateurs qui élaborent les catégories de chômeurs et de chômage évacuent la question des machines car pour eux, note Christian Topalov, " le progrès du machinisme et l'élargissement de l'échelle de la production concourent à réduire l'instabilité de l'emploi ${ }^{58} "$. Alfred Marshall, en Angleterre comme Davis R. Dewey aux États-Unis, affirment ainsi que les grandes machines coûteuses permettent de régulariser la production et donc l'emploi, faisant ainsi disparaître les phases de chômage chronique. Dans ce cadre, c'est d'abord la persistance du travail manuel dans les petites industries qui est la cause principale de la misère ouvrière, alors que la grande industrie mécanisée fait espérer l'apparition d'un emploi plus stable et régularisé.

La question des machines et de leur impact ressurgit périodiquement à chaque moment de crise socio-économique, suscitant débats et controverses sur les meilleurs moyens de réguler leurs effets. Vers 1880, W. Moody

55. Moses, 2009.

56. Salais, Baverez, Reynaud, 1986.

57. Rapport sur la question du chômage, présenté au nom de la commission permanente du Conseil supérieur du travail, 1896, p. viii et 323.

58. Topalov, 1994. 
suggère par exemple de remédier aux effets néfastes $\mathrm{du}$ " machinisme " par la réduction de la durée du travail. En Angleterre, le journaliste libéral Hobson, pourfendeur de l'impérialisme, discute les effets négatifs des machines sur l'emploi au moyen de statistiques ${ }^{59}$. En France, à l'occasion de l'exposition de 1889, Charles Gide, l'un des principaux théoriciens de l'économie sociale, décrit le "déploiement énorme de puissance productive, [qui] donne plus de satisfaction à l'orgueil de l'homme qu'à ses véritables besoins ». Il stigmatise les " redoutables appareils de l'industrie de notre temps " car "l'ouvrier laisse quelquefois dans leurs engrenages son bras ou sa jambe ", mais il affirme en même temps le caractère inévitable du processus ${ }^{60}$. Contre les solutions socialistes et collectivistes il propose de faire des coopératives de consommation des outils permettant de domestiquer la surproduction industrielle au service de l'émancipation du prolétariat.

L'impact des technologies est également discuté par les mouvements ouvriers de plus en plus structurés en syndicats et en partis de masse. En Angleterre, le Trade-Union Congress naît à Manchester dès 1868, en France les syndicats sont autorisés en 1884. Leurs effectifs connaissent une forte croissance - s'il n'y a par exemple que 671000 ouvriers syndiqués en France en 1911, ils sont respectivement 2,6 et 3,4 millions en Allemagne et en Grande-Bretagne. Au sein de ces organisations, les nouvelles technologies sont de plus en plus acceptées comme inéluctables, voire investies de nouveaux espoirs. La " classe ouvrière » toujours en construction à la fin du siècle s'identifie de plus en plus à l'homme prométhéen, c'est moins l'intelligence et les savoir-faire manuels qui sont exaltés que la puissance mécanique domptée par l'homme. L'ouvrier « brandisseur de marteau » envahit d'ailleurs l'imagerie du mouvement ouvrier, et l'expérience du travail tend de plus en plus à s'identifier à celle du contact viril avec la machine plutôt qu'à la maîtrise d'un savoir-faire complexe. Face aux innovations, les représentants syndicaux exigent des compensations salariales ou une diminution du temps de travail. Alors que la machine est devenue l'auxiliaire de l'ouvrier plutôt que son ennemi, les négociations pour les apprivoiser prennent le pas sur les protestations collectives violentes ${ }^{61}$. Dans sa remarquable synthèse, Alain Dewerpe estime que dans les syndicats des ouvriers de métier

59. Moody, 1878 ; Hobson, 1893.

60. Gide, 2001, p. 133.

61. Van Lente, 1993, p. 56. 
"les nouvelles technologies sont acceptées ", elles sont même de plus en plus recherchées « dès lors qu'elles peuvent apparaître comme un moyen de réduire l'usure au travail et de promouvoir une nouvelle autonomie ouvrière $^{62}$ ». Même le syndicalisme révolutionnaire de la fin du XIX ${ }^{e}$ siècle, qui prône le "sabotage ", respecte désormais l'outil de travail. Comme l'écrivait encore Eric Hobsbawm à la suite des époux Webb, «le but qui consistait à empêcher l'introduction de machines indésirables, céda alors le pas, avec l'arrivée de la mécanisation totale, à d'autres visées. Le nouvel objectif était de " capturer les machines pour les mettre aux mains de travailleurs qui bénéficiaient des conditions et des règles syndicales, tout en prenant toutes mesures utiles pour limiter le chômage technologique ${ }^{63}$ ".

Après 1890, les concentrations industrielles et la mécanisation s'accentuent en bouleversant les équilibres internes du monde du travail et en donnant naissance à un nouveau savoir ${ }^{64}$. Alors que les tisseurs et tisserands sont pris comme nous l'avons vu dans une course à la productivité, les anciens puddleurs sont remplacés par l'invention du convertisseur Bessemer et les fileurs deviennent de simples surveillants des self-acting mule. Ces transformations, durement ressenties par de nombreux ouvriers de métiers, entraînent selon l'historien Gérard Noiriel une véritable " crise des valeurs dans la classe ouvrière ${ }^{65}$ ». Dans la plupart des pays européens pourtant, l'outillage change lentement. Dans de nombreuses régions les paysans labourent toujours avec l'aide d'outils en bois et de leurs animaux ; beaucoup d'ouvriers de métier et d'artisans continuent de travailler dans de petites structures où la mécanisation et la discipline peinent à s'imposer. L'usine elle-même reste souvent un univers complexe composé d'un agrégat d'ateliers où la part du travail manuel demeure très importante ${ }^{66}$. Même si la proportion des ouvriers nés de la technique industrielle, comme dans la sidérurgie ou les industries mécaniques, s'accroît indéniablement, ils sont très loin de représenter la majorité des effectifs. Pour ces nouveaux groupes, le changement des méthodes productives devient une réalité familière, même si la crainte et la suspicion persistent.

62. Dewerpe, 1989 , p. 106.

63. Hobsbawm, 1952 ; Webb, 1897, chap. VIII : «New processes and machinery », p. 411.

64. Moss, 1985.

65. Noiriel, 1986, p. 84.

66. Lefebvre, 2003. 
Plutôt que d'évoquer une figure ouvrière abstraite qui n'existe pas, il convient donc d'être attentif à la pluralité des situations et à la diversité des expériences. L'« organisation scientifique du travail ", ou «scientific management ", s'étend peu à peu dans un espace transnational en s'appuyant sur le développement de techniques de contrôle du temps et de l'espace. Mais ce qu'on appelle la "rationalisation » a suscité depuis les années 1970 de très nombreux travaux qui montrent la diversité des formes prises par ces nouvelles techniques de production selon les pays, les branches, les entreprises, à l'intersection entre l'utopie technique de maitrise du monde des ingénieurs et les négociations et bricolages sociotechniques incessants des acteurs ouvriers. D'abord portée aux États-Unis par l'ingénieur Taylor à la fin du XIX ${ }^{e}$ siècle, le taylorisme s'étend ensuite en Europe : ses ouvrages sont traduits en français au début du $\mathrm{xx}^{\mathrm{e}}$ siècle, et Louis Renault le rencontre dès 1911. Même si les premières tentatives d'introduction suscitent des oppositions - quelques grèves aux États-Unis et une chez Renault lorsqu'est importée la méthode du chronométrage en 1913 -, les conflits restent dans l'ensemble limités ${ }^{67}$.

La Première Guerre mondiale et les recompositions des années 1920-1930 accélèrent encore le basculement vers un nouveau régime de productivité industrielle fondé sur l'usage intensif de puissantes machines, et l'adoption parallèle de nouvelles formes d'organisation du travail. La mobilisation massive des conscrits en 1914 conduit en effet à l'adoption de technologies plus productives, et à l'embauche de femmes pour les conduire alors que partout s'accélère le tournant taylorien des pays industrialisés ${ }^{68}$. L'entre-deux-guerres est également une période d'intenses transformations techniques et organisationnelles pour les mondes du travail alors que l'électrification s'accroît. Le mot productivisme apparaît d'ailleurs à cette époque pour désigner le système économique dans lequel l'accroissement de la production devient l'objectif premier, inaugurant une nouvelle trajectoire de rationalisation et d'automatisation du travail qui ne cesse de s'accentuer ensuite.

67. Fridenson, 1987 ; l'historiographie du taylorisme et du travail à la chaîne s'est beaucoup développée en insistant sur les décalages constants entre l'organisation prescrite du travail et les pratiques réelles, cf. Michel, 2007.

68. Downs, 2002. 


\section{Sources}

Archives de l'État de Liège (AEL) :

- Fonds hollandais, 803 : rapports périodiques de police de l'arrondissement de Verviers, janvier 1819.

- Fonds hollandais, 3521 : commune de La Reid (canton de Spa).

- Fonds hollandais, 3539 : états des fabriques et ateliers à Verviers au

31 décembre 1819.

Archives communales de Verviers, Farde 39 : Indemnisation Coumont-Grosfils, réclamation $n^{\circ} 16$.

Borgnis Giuseppe Antonio, Traité complet de mécanique appliquée aux arts, contenant l'exposition méthodique des théories et des expériences les plus utiles pour diriger le choix, linvention, la construction et l'emploi de toutes les espèces de machines, Bachelier, Paris, 1820.

Briancourt Mathieu, Visite au Phalanstère, Librairie phalanstérienne, Paris, 1848.

Costaz Claude A., Essai sur l'administration de l'agriculture, du commerce, des manufactures et des subsistances, suivi de l'historique des moyens qui ont amené le grand essor pris par les arts depuis 1793 jusquien 1815, Huzard, Paris, 1818.

Engels Friedrich et Marx Karl, Le Manifeste du parti communiste, trad. de Laura Lafargue, Le Manifeste communiste aujourd'hui, Éditions de l'Atelier, Paris, 1998.

Etzler John A., The Paradise within the Reach of all Men without Labor, by Powers of Nature and Machinery. An Address to all Intelligent Men, in two parts, Etzler and Reinhold, Pittsburgh, 1833.

Gide Charles, "De la coopération et des transformations qu'elle est appelée à réaliser dans l'ordre économique. Discours d'ouverture du Congrès international des Sociétés coopératives de consommation tenu à Paris, au Palais du Trocadéro, le 8 septembre 1889 ", dans Charles Gide. Coopération et économie sociale (1886-1914), L'Harmattan, Paris, 2001, p. 133.

Hobson John A., "The influence of machinery upon employment", Political Science Quarterly, vol. 8, n 1, 1893, p. 97-123.

Michelet Jules, Le Peuple, Flammarion, Paris, 1974 [1846].

Michelet Jules, Euvre complète, Flammarion, Paris, 1893-1898, vol. 24 : "Histoire du XIX ${ }^{\mathrm{e}}$ siècle ", Préface.

Moody William Godwin, Our Labor Difficulties: The Cause, and the Way Out; Including the Paper on the Displacement of Labor by Improvements in Machinery, 1878.

Notice sur une nouvelle machine à tondre les draps appelée tondeuse ou Forces Hélicoïdes, Vve Courcier, Paris (s. d.). 
Proudhon Pierre J., Système des contradictions économiques ou misère de la philosophie, Paris, 1846.

Rapport sur la question du chômage, présenté au nom de la commission permanente du Conseil supérieur du travail, Imprimerie nationale, Paris, 1896.

ReEs Abraham, The Cyclopedia, 45 vol., Longman, Hurst, Rees, Orme \& Brown, Londres, 1819, vol. 38.

Simond Louis, Voyage d'un français en Angleterre pendant les années 1810 et 1811 avec des observations sur l'état politique et moral, les arts et la littérature, Treuttel et Wurtz, Paris, 1816, 2 vol., t. 2.

Ure Andrew, The Philosophy of Manufactures: Or, An Exposition of the Scientific, Moral, and Commercial Economy of the Factory System, Charles Knight, Londres, 1835.

\section{Bibliographie}

Adams Henry, "The Dynamo and the Virgin (1900) ", The Education of Henry Adams, Oxford University Press, Oxford, 1999.

BeEcher Jonathan, Fourier. The Visionary and His World, University of California Press, Berkeley, 1990.

Belhoste Bruno et Chatzis Konstantinos, "From technical corps to technocratic power: French state engineers and their professional and cultural universe in the first half of the 19th century", History and Technology, n' 23, 2007, p. 209-225.

Benoit Serge, "L'introduction des métiers Northrop ", dans Favier René, Gayot Gérard, KLeIn Jean-François, Terrier Didier, Woronoff Denis (dir.), Tisser l'histoire. L'industrie et ses patrons, $X V I^{e}-X X^{e}$ siècles, Presses universitaires de Valenciennes, Valenciennes, 2009, p. 85-96.

Berg Maxine, The Machinery Question and the Making of Political Economy 18151848, Cambridge University Press, Cambridge, 1982.

Binfield Kevin (dir.), Writings of the Luddites, The Johns Hopkins University Press, Baltimore/Londres, 2004.

Bourdeau Vincent, Jarrige François et Vincent Julien, Les Luddites. Bris de machine, économie politique et histoire, Éditions Ère, Maisons-Alfort, 2006.

CARnino Guillaume, "Les transformations de la technologie : du discours sur les techniques à la "technoscience” ", Romantisme, n 150, 2010, p. 75-84.

Claeys Gregory, "John Adolphus Etzler, technological utopianism, and British socialism: the tropical emigration society's Venezuelan mission and its social context, 1833-1848 ", The English Historical Review, vol. 101, n 399, 1986, p. 351-375. 
Cohen Yves, Organiser à l'aube du taylorisme, La pratique d'Ernest Mattern chez Peugeot (1906-1919), Presses universitaires de Franche-Comté, Besançon, 2001.

Daumas Maurice (dir.), Histoire générale des techniques, Tome 3. L'expansion du machinisme, 1725-1860, PUF, coll. Quadrige, Paris, 1996 [1968].

Delrée Henri et HÉlin Étienne, "Contre les machines, pour le plein-emploi ? Un réquisitoire de Laurent-François Déthier (1757-1843) ", Bulletin de la Société royale du Vieux Liège, t. XI, n 235-237-238, 1986-1987, p. 253-263.

Dewerpe Alain, Le Monde du travail en France (1800-1950), Armand Colin, Paris, 1989.

Downs Laura Lee, L'Inégalité à la chaîne. La division sexuée du travail dans l'industrie métallurgique en France et en Angleterre, Albin Michel, Paris, 2002.

Feller Irwin, "The draper loom in New England textiles, 1894-1914: a study of diffusion of an innovation", The Journal of Economic History, t. xxvi, 1966, $\mathrm{n}^{\circ} 3$, p. 320-347.

Fohal J., Les Événements de 1830 à Verviers et aux environs, Verviers, 1930.

Fridenson Patrick, "Un tournant taylorien de la société française (1904-1918) », Annales ESC, n 5, sept.-oct. 1987, p. 1031-1060.

GaYoт Gérard, "La classe ouvrière saisie par la révolution industrielle à Verviers, 1800-1810 », Revue du Nord, t. 84, n 347, 2002, p. 633-666.

GAYот Gérard, "Les tondeurs dans les manufactures européennes au XVIII ${ }^{\mathrm{e}}$ siècle », Le Culture della Technica, Archivio Storico Amma, nouvelle série 14, 2/2002, p. 1-36.

GАуот Gérard, «Frontières, barrières douanières et métamorphoses des territoires industriels entre Meuse et Elbe (1750-1815) ", Revue du Nord, t. 85, n 352, 2003, p 781-808.

Godin Benoit, Innovation Contested. The Idea of Innovation Over the Centuries, Routledge, Londres 2015.

Godley Andrew, "Selling the sewing machine around the world: Singer's international marketing strategies, 1850-1920", Enterprise and Society, 2006, p. 266-314.

Griffin Carl J., The Rural War: Captain Swing and the Politics of Protest, Manchester University Press, Manchester, 2012.

Guillerme Jacques et Sebestik Jan, "Les commencements de la technologie ", Thalès, t. 12, 1968, p. 1-72, rééd. dans Documents pour l'histoire des techniques, $\mathrm{n}^{\circ} 14,2007$, p. 50-121.

Halleux Robert, Le Savoir de la main. Savants et artisans dans l'Europe pré-industrielle, Armand Colin, Paris, 2009.

Hilaire-PÉrez Liliane, L'Invention technique au siècle des Lumières, Albin Michel, Paris, 2000. 
Hilaire-Pérez Liliane, La Pièce et le geste. Artisans, marchands et savoir technique à Londres au XVIII siècle, Albin Michel, Paris, 2013.

Hовзвашм Eric, "Machine breakers », Past \& Present, n 1, 1952, p. 57-70.

Hornborg Alf, The Power of the Machine: Global Inequalities of Economy, Technology, and Environment, AltaMir, Walnut Creek, 2001.

JarRige François, Au temps des tueuses de bras. Les bris de machines à l'aube de l'ère industrielle, PUR, Rennes, 2009.

JARRIGE François, "Le travail discipliné : genèse d'un projet technologique au $\mathrm{XIX}^{\mathrm{e}}$ siècle ", Cahiers d'histoire. Revue d'histoire critique, $\mathrm{n}^{\circ} 110$, octobre-décembre 2009, p. 99-116.

JARRIGE François, "Les tondeurs européens à l'épreuve des mécaniques : approche comparée des négociations sociotechniques dans l'industrie lainière (1750-1850) ", dans Maitte Corine, Minard Philippe et Oliveira Mathieu de (dir.), La Gloire de l'industrie. Faire de l'histoire avec Gérard Gayot, PUR, Rennes, 2012, p. 279-298.

Jarrige François (dir.), Dompter Prométhée. Technologies et socialismes à l'âge romantique (1820-1870), Presses universitaires de Franche-Comté, Besançon, 2016.

JARRIGE François, "L'invention de "l'ouvrier machine" : esclave aliéné ou pure intelligence au début de l'ère industrielle? ", L'Homme et la société. Revue internationale de recherches et de synthèses en sciences sociales, $\mathrm{n}^{\circ} 205$ : "L'hommemachine I- Le travailleur machine ", 2017, p. 27-52.

JARrige François, "Work, skill and technology ", dans Thompson Victoria E. (dir.), The Cultural History of Work in the Age of Empire (1800-1920), Arizona State University, Tempe, 2018, p. 85-98.

Jarrige François et VRIGnon Alexis, Face à la puissance. Une histoire des énergies alternatives à l'âge industriel, La Découverte, Paris, 2020.

Johnson C. H., The Life and Death of Industrial Languedoc (1700-1900), Oxford University Press, New York/Oxford, 1995.

Kisch H., "The Textile industries in Silesia and the Rhineland: a comparative study in industrialization", dans Kriedte Peter, Medick Hans, Schlumbhom Jurgen (dir.), Industrialization before Industrialisation. Rural Industry in the Genesis of Capitalism, CUP/MSH, 1980, Cambridge/Paris, p. 178-200.

Lebrun P., L'Industrie de la laine à Verviers pendant le XVIII siècle et le début du $X I X X^{e}$ siècle. Contribution à l'étude des origines de la révolution industrielle, Faculté de philosophie et Lettres, Liège, 1948.

Lefebvre Philippe, L'Invention de la grande entreprise. Travail, hiérarchie, marché, France, fin XVIII' -début XX' siècle, PUF, Paris, 2003.

Lejaer Jean, "Histoire de la ville de Verviers. Période hollandaise ", Bull. Soc. Verviétoise d'Archéologie et d'Histoire, t. vII, 1906, p. 4-320. 
Leménorel Alain, Désert Gabriel, Dupré Philippe, Lecherbonnier Yannick, RÉAL Emmanuelle, "Hydraulique, vapeur et industrialisation au XIX siècle : la "voie normande" ", Cahier des Annales de Normandie, n 25, 1993, p. 7-22.

LE Roux Thomas, "Les puissances vives soumises aux forces mortes. Hygiénistes, corps ouvriers et machines au XIX ${ }^{\mathrm{e}}$ siècle en France (1800-1870) ", dans Guignard Laurence, Raggi Pascal, Thévenin Étienne (dir.), Corps et machines à l'âge industriel, XIX'-XX siècles, PUR, Rennes, 2011, p. 259-272.

MacLeod Christine, Heroes of Invention. Technology, Liberalism and British Identity (1750-1914), Cambridge University Press, Cambridge, 2007.

Malm Andreas, Fossil Capital: The Rise of Steam Power and the Roots of Global Warming, Verso, Londres, 2016.

Manuel F. R., “The Luddite Movement in France”, Journal of Modern History, juin 1938, p. 180-211.

Marx Leo, The Machine in the Garden: Technology and the Pastoral Ideal in America, Oxford University Press, Oxford, 2000 [1964].

Michel Alain P., Travail à la chaîne. Renault 1898-1947, ETAI, Boulogne-Billancourt, 2007.

Moses Julia, "Accidents at work, security and compensation in industrialising Europe: the cases of Britain, Germany and Italy, 1870-1925”, Annual Review of Law and Ethics, $\mathrm{n}^{\circ} 17,2009$, p. 237-258.

Moss Bernard H., Aux origines du mouvement ouvrier français, Presses universitaire de Franche-Comté, Besançon, 1985.

Mouchel Charles, "Étude sur les origines du tondage des draps ", Bulletin des travaux de la société industrielle d'Elbeuf, 1885, n 1, p. 168-189.

Noiriel Gérard, Les Ouvriers dans la société française, XIX $-X X^{e}$ siècle, Seuil, Paris, 1986.

Nye David E., American Technological Sublime, The MIT Press, Cambridge, 1994.

Perrot Michelle, "Machines à coudre et travail à domicile ", Le Mouvement social, $\mathrm{n}^{\circ} 105,1978$, p. 161-164.

Perrot Michelle, "Femmes et machines au Xix siècle ", dans Les Femmes ou les silences de l'histoire, Flammarion, Paris, 1998.

PEYrière Monique, Recherches sur la machine à coudre, 1830-1889, EHESS, Paris, 1990.

Pilisi Jean, "Le tondage du drap dans l'histoire des techniques », L'Industrie textile, 1954-1956 (17 articles, 76 p.).

Pilisi Jean, "Le chevalier Cochelet et la machine à tondre les draps (17881858) ", Chambre et Sénat: revue d'études et d'informations, 1957.

Randall Adrian, Before the Luddites: Custom, Community and Machinery in the English Woollen Industry, 1776-1809, Cambridge University Press, Cambridge, 1991. 
Rule John, "The property of skill in the period of manufacture", dans JoyCE Patrick (dir.), The Historical Meanings of Work, Cambridge University Press, Cambridge, 1987, p. 99-118.

Riot-Sarcey Michèle, Le Procès de la liberté. Une histoire souterraine du XIX' siècle en France, La Découverte, Paris, 2016.

Salais Robert, Baverez Nicolas, Reynaud Bénédicte, L'Invention du chômage, PUF, Paris, 1986.

Samuel Raphael, "Workshop of the World: Steam Power and Hand Technology in Mid Victorian Britain", History Workshop Journal, n 3, 1977, p. 6-72.

Thompson Edward P., The Making of the English Working Class, Penguin Books, Toronto, 1991 [1963].

Topalov Christian, Naissance du chômeur (1880-1910), Albin Michel, Paris, 1994.

Tresch John, The Romantic Machine. Utopian Science and Technology after Napoleon, The University of Chicago Press, Chicago, 2012.

VAn Lente Dick, "The critique of industrial technology in the Netherlands and other Western Countries in the nineteenth century", dans Christensen Dan Ch. (dir.), European Historiography of Technology, Odense University Press, Odense, 1993, p. 55-67.

VAn NeCK Anne, Les Débuts de la machine à vapeur dans l'industrie Belge, 18001850, Palais des Académies, Bruxelles, 1979.

Viallaneix Paul, « Michelet, machines, machinisme ", Romantisme, n 23, 1979, p. 3-15.

Von Tunzelmann Nick, Steam power and British industrialization to 1860, Clarendon Press, Oxford, 1978.

Verley Patrick, L'Échelle du monde. Essai sur l'industrialisation de l'Occident, Gallimard, Paris, 1997.

Weвв Béatrice et Sydney, Industrial Democracy, Longman, Londres, 1897.

\section{L'auteur}

François Jarrige est maître de conférences en histoire contemporaine à l'université de Bourgogne (LIR3S UMR 7366 CNRS) et membre de l'Institut universitaire de France. Il s'intéresse à l'histoire du travail, des techniques et de l'environnement. II a publié récemment, avec Alexis Vrignon, Face à la puissance. Une histoire des énergies alternatives à l'âge industriel, Paris, La Découverte, 2020, et, avec Thomas Le Roux, La Contamination du monde. Une histoire des pollutions à l'âge industriel, Paris, Seuil, coll. « L'univers historique », 2017. Contact : francois. jarrige@u-bourgogne.fr 